\title{
Comment mesurer le débit en collecteur d'assainissement en remplaçant un capteur de vitesse par un deuxième capteur de niveau d'eau?
}

Sandra Isel ${ }^{1}$, José Vazquez ${ }^{2}$, Martin Fischer ${ }^{2}$, Maxence Graebling ${ }^{2}$, Jonathan Wertel ${ }^{1}$, Claude Joannis ${ }^{3}$, Matthieu Dufresne ${ }^{2}$

${ }^{1}$ 3D EAU, 4 rue Boussingault 67000 Strasbourg - courriel : sandra.isel@3deau.fr, jonathan.wertel@3deau.fr

${ }^{2}$ Ecole Nationale du Génie de l'Eau et de l'Environnement de Strasbourg, Laboratoire ICube (Université de Strasbourg, CNRS, INSA de Strasbourg, ENGEES - adresse: ICube, 2 rue Boussingault $67000 \quad$ Strasbourg $\quad$ - courriels : jose.vazquez@engees.unistra.fr, martin.fischer@engees.unistra.fr, mgraebli@engees.eu, matthieu.dufresne@engees.unistra.fr

${ }^{3}$ Institut Français des Sciences et Technologies des Transports, de l'Aménagement et des Réseaux (IFSTTAR), Laboratoire Eau et Environnement - adresse : IFSTTAR Centre de Nantes, Route de Bouaye 44344 Bouguenais - courriel : claude.joannis@ifsttar.fr

English title: how to measure the flow in sewers by replacing a velocity sensor by a second water level sensor?

Mots-clés : assainissement, collecteur, débit, hauteur d'eau, mesure

Keywords: sewerage, pipe, discharge, water depth, measurement

Résumé

La méthode h2Q présentée dans cet article permet la détermination du débit en collecteur uniquement à partir de deux mesures de niveau d'eau réalisées à l'amont et à l'aval d'un tronçon de quelques dizaines de mètres. Ceci en fait une méthode peu coûteuse à l'investissement et à l'exploitation puisqu'elle ne met en œuvre que des capteurs sans contact qui ne nécessitent que peu de maintenance par rapport à des capteurs immergés. La méthode $\mathrm{h} 2 \mathrm{Q}$ repose sur la génération à partir du modèle de la courbe de remous d'un tableau de valeurs $\left(h_{1}, h_{2}, Q\right)$ mettant en relation la hauteur amont, la hauteur aval et le débit. Mise en œuvre sans calage, elle permet l'évaluation du débit avec une incertitude de l'ordre de $30 \%$. Un calage permet de réduire, l'incertitude à environ $15 \%$, soit une valeur comparable aux autres technologies disponibles sur le marché lorsqu'elles sont mises en place conformément aux règles de l'art. Une validation de la méthode sur deux sites de mesure grandeur nature est présentée afin de démontrer son caractère opérationnel.

\section{Abstract}

This article introduces the $\mathrm{h} 2 \mathrm{Q}$ method. This method allows the determination of the discharge in sewer pipe thanks to two water level measurements. Investment and operating costs are limited for this technique because it only involves contactless sensors. A backwater curve model is used to generate a three column table $\left(h_{1}, h_{2}, Q\right)$ involving the upstream water depth, the downstream water depth and the discharge. Without calibration, this method leads to an uncertainty evaluated to $30 \%$. With calibration, the uncertainty decreases to about $15 \%$, which is comparable to the other technologies available on the market (when they are installed in good conditions). Two on-site applications are presented in order to validate the method and demonstrate that it is operational. 


\section{Introduction}

Il existe plusieurs méthodes pour mesurer en continu un débit en collecteur d'assainissement. La plus universelle est basée sur une évaluation de la vitesse moyenne dans une section et de la surface mouillée de cette section. Diverses technologies de mesures de vitesse sont disponibles sur le marché: capteurs Doppler continus, profilomètres, cordes de vitesse, radars de surface... Lorsqu'elles sont mises en œuvre conformément aux règles de l'art (normes ISO 15769, ISO 6416), ces techniques permettent d'obtenir une incertitude sur le débit d'environ $10 \%$ à $20 \%$. Toutefois ces techniques sont relativement coûteuses, en investissement comme en maintenance.

Il est plus économique d'évaluer un débit à partir d'une hauteur d'eau (de préférence sans contact) sans mesure de vitesse. Pour cela on peut mettre en place dans le collecteur un aménagement permettant de créer localement des conditions d'écoulement (passage en régime torrentiel) garantissant une relation univoque hauteur / débit: des seuils ou parfois des canaux de type Venturi (manchons avec contraction). Cette méthode est cependant rarement mise en place de façon continue du fait des forts risques d'encrassement ou d'obstruction. Elle est plus adaptée pour des campagnes temporaires.

On peut aussi postuler l'existence d'une relation hauteur débit dans un collecteur non aménagé dans ce but. Une telle relation existe effectivement en régime permanent et surtout uniforme, c'est-à-dire avec une ligne d'eau parallèle au radier. On peut alors appliquer une formulation de type Manning Strickler, le cas échéant étalonnée par jaugeage. Mais en pratique on est suffisamment éloigné du régime permanent uniforme, notamment dans les réseaux présentant des pentes faibles (quelques dixièmes de pourcents) pour que la notion même de relation univoque entre hauteur et débit perde toute validité : des phénomènes d'influence aval peuvent imposer des valeurs de hauteur très différentes pour une même valeur de débit. La figure 1 illustre ainsi le cas d'une station de mesure pour laquelle aucune relation univoque n'existe entre la hauteur mesurée et le débit circulant dans la canalisation. Si on utilisait la relation de Manning-Strickler pour cette station de mesure ainsi qu'illustré par la courbe verte pointillée sur la figure 1 (avec une rugosité de Strickler de $70 \mathrm{~m}^{1 / 3} / \mathrm{s}$ ), on commettrait des erreurs comprises entre $50 \%$ et $810 \%$ (en moyenne $340 \%$ ). Si on utilisait une courbe d'étalonnage (en rouge sur la figure 1), on commettrait des erreurs comprises entre $0 \%$ et $140 \%$. La figure 1 montre bien que cette courbe ajustée n'est pas représentative du fonctionnement hydraulique de cette station de mesure.

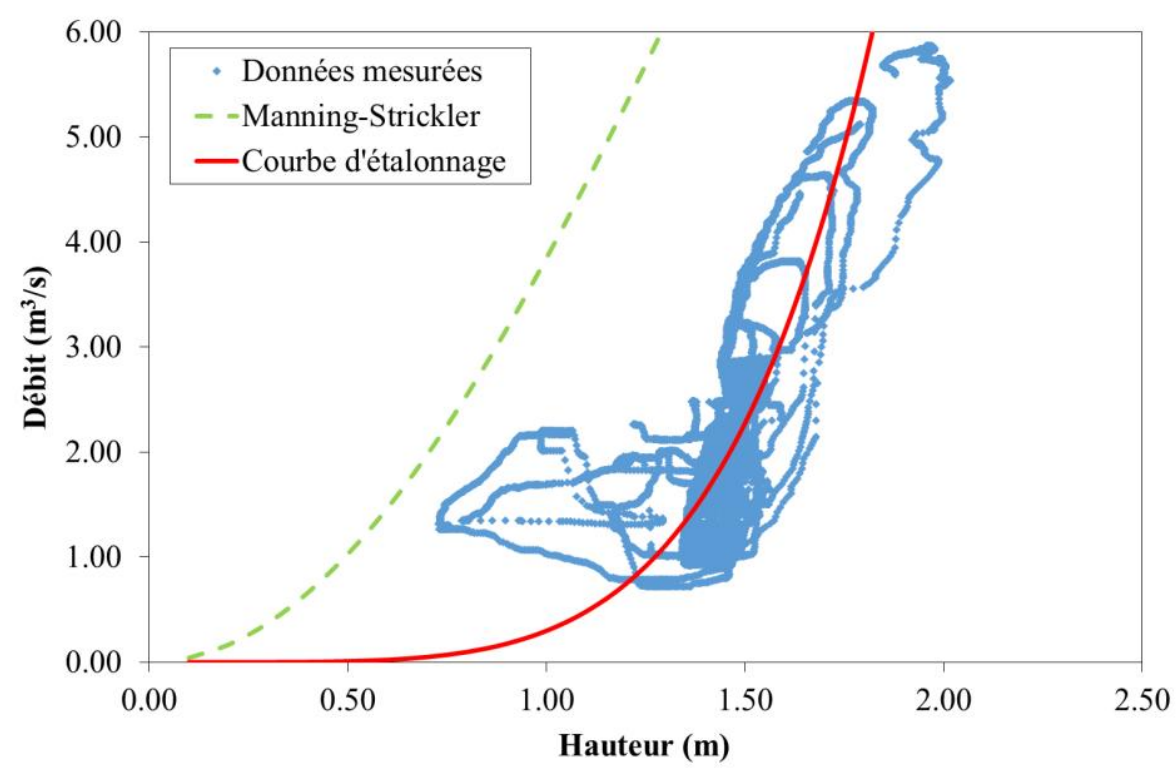

Figure 1. Exemple d'une relation hauteur / débit non univoque. 
L'utilisation de la relation de Manning-Strickler nécessite en effet de vérifier au préalable que la hauteur d'eau est uniforme le long du collecteur. Lorsque le régime uniforme n'est pas garanti, on ne peut plus considérer que la pente de la ligne d'eau est égale à celle du radier mais on peut la mesurer à l'aide de deux capteurs suffisamment éloignés pour obtenir une précision suffisante sur le dénivelé. Cette méthode est notamment préconisée en rivière ( «station à double échelle » de la Charte qualité de l'hydrométrie). Le deuxième capteur de hauteur se substitue en quelque sorte à un capteur de vitesse.

La méthode h2Q décrite dans cet article est une variante de cette méthode. Elle est basée sur le calcul complet des courbes de remous plutôt que sur la pente moyenne de la ligne d'eau (elle évite ainsi l'approximation de la ligne d'eau par une droite). Cette méthode permet donc de prendre parfaitement en compte les influences aval et cumule les avantages de mesures de hauteurs d'eau (sans contact) avec un large domaine de validité. En effet, non seulement elle prend en compte les phénomènes d'influence aval (contrairement à la relation de Manning-Strickler) mais en plus elle est valide dans le cas où la ligne d'eau n'est pas plane (contrairement à la méthode basée sur la pente moyenne de la ligne d'eau).

Les objectifs de cet article sont d'exposer le principe de cette méthode puis d'en expliciter la mise en œuvre. Enfin une troisième partie sera consacrée à la validation de la méthode h2Q sur site.

\section{Description de la méthode}

\subsection{Principe}

La méthode $\mathrm{h} 2 \mathrm{Q}$ consiste à mesurer la différence de niveau d'eau entre deux regards distants de quelques dizaines à quelques centaines de mètres puis à calculer le débit à partir de l'évaluation de la perte de charge (linéaire) entre les deux regards par le biais de l'équation de la courbe de remous (Hager 1999). La méthode est également capable de prendre en compte des effets transitoires forts ; dans ce cas, elle utilise les équations de Saint-Venant (Isel 2014). Ce principe est illustré sur la figure 2. Une description plus détaillée de la méthode est fournie dans les références suivantes : Isel et al 2014, Isel et al 2016.

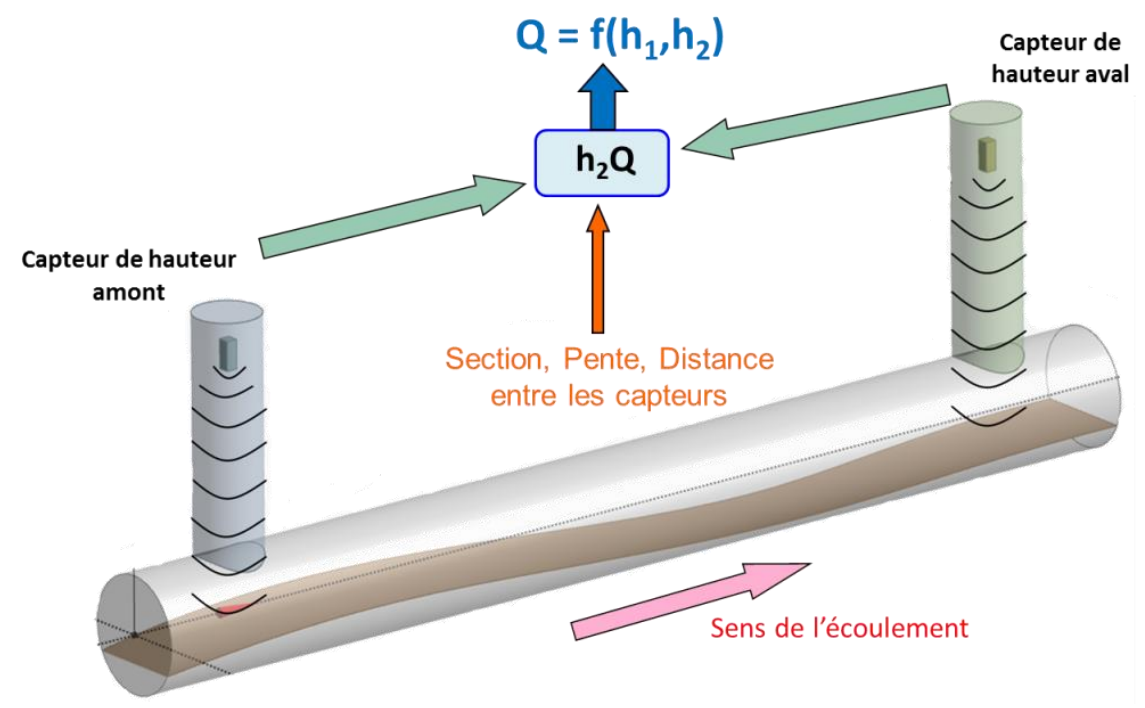

Figure 2. Principe de la méthode h2Q.

1.2 Données d'entrée 
Les données d'entrée nécessaires à cette méthode sont les caractéristiques de la canalisation, à savoir : sa section, sa taille, sa pente ainsi que la distance entre les deux capteurs. La rugosité, valant généralement autour de $70 \mathrm{~m}^{1 / 3} / \mathrm{s}$ en réseau d'assainissement, peut également être un paramètre permettant d'affiner le calcul (voir plus loin la comparaison avec et sans calage). La méthode est applicable à toutes les formes de collecteur (circulaire, ovoïde, fer-à-cheval, rectangulaire, forme quelconque...). Dans le cas d'une forme complexe, un relevé géomètre peut s'avérer nécessaire. La méthode h2Q est également applicable lorsque le collecteur fonctionne en charge.

\subsection{Résultats}

La méthode fournit une relation entre les deux hauteurs mesurées dans les deux regards et le débit circulant dans le collecteur. Cette relation peut être présentée sous la forme d'un tableau à 3 colonnes $\left(h_{1}, h_{2}, Q\right)$ ou d'une fonction mathématique $Q=f\left(h_{1}, h_{2}\right)$. Dans ce tableau ou cette fonction, plusieurs couples $\left(h_{1}, h_{2}\right)$ peuvent correspondre au même débit $Q$ du fait des influences aval.

\subsection{Précision}

Les sources d'incertitude de la méthode h2Q sont les suivantes (classement des plus importantes aux plus faibles)

- L'incertitude de mesure sur les hauteurs d'eau : elle est due d'une part à l'incertitude sur la mesure de niveau en elle-même (liée à la technologie de mesure ainsi qu'à la surface libre) mais aussi au zéro du capteur (effectué par rapport au radier du collecteur). On estime cette incertitude à environ une dizaine de millimètres. En propageant cette incertitude sur le débit, on estime que l'incertitude de mesure devient inférieure à $20 \%$ dès que la différence de niveau entre les deux mesures atteint environ $5 \mathrm{~cm}$; elle devient inférieure à $10 \%$ dès environ $10 \mathrm{~cm}$ de différence (Isel 2014). Précisons que ces valeurs ne constituent que des ordres de grandeur et qu'une évaluation plus fine peut être effectuée au cas par cas en fonction des caractéristiques de la station de mesure considérée.

- L'incertitude sur la rugosité : la rugosité hydraulique est calculée à partir de la rugosité de la conduite (considérée égale à $70 \mathrm{~m}^{1 / 3} / \mathrm{s}$ ) selon l'approche de Camp (prise en compte des frottements plus importants aux petits remplissages). En réalité, la rugosité de la conduite peut différer de cette valeur de $70 \mathrm{~m}^{1 / 3} / \mathrm{s}$. On peut estimer l'incertitude sur cette valeur à environ $20 \%$ à $30 \%$. Cette incertitude est réduite dans le cas où la méthode h2Q est calée sur des mesures de références (de l'ordre de $10 \%$ si la mesure servant de calage est de qualité).

- L'incertitude de l'équation de la courbe de remous utilisée pour évaluer le débit à partir des deux hauteurs d'eau : environ 5\%.

- L'incertitude d'ajustement de la fonction mathématique $Q=f\left(h_{1}, h_{2}\right)$ ou d'interpolation si on utilise une représentation tabulaire : quelques pourcents au maximum.

- L'incertitude sur les données d'entrée (pente, diamètre, distance entre les regards): généralement insignifiante par rapport aux autres. Notons cependant que la pente n'est pas toujours connue avec une grande précision et qu'une vérification sur site peut s'avérer nécessaire.

En combinant toutes ces sources d'incertitude et en les supposant indépendantes les unes des autres, on peut estimer l'incertitude à environ $30 \%$ pour la méthode h2Q sans calage et environ $15 \%$ pour la méthode h2Q avec calage (lorsque la méthode est mise en place dans des conditions adaptées ainsi qu'expliqué plus loin).

\section{Mise en œuvre}


La mise en œuvre de la méthode h2Q peut être synthétisée sous la forme de l'organigramme suivant. Les étapes principales sont explicitées ci-dessous. Pour un site donné, elle demande quelques jours de travail pour être mise en œuvre.

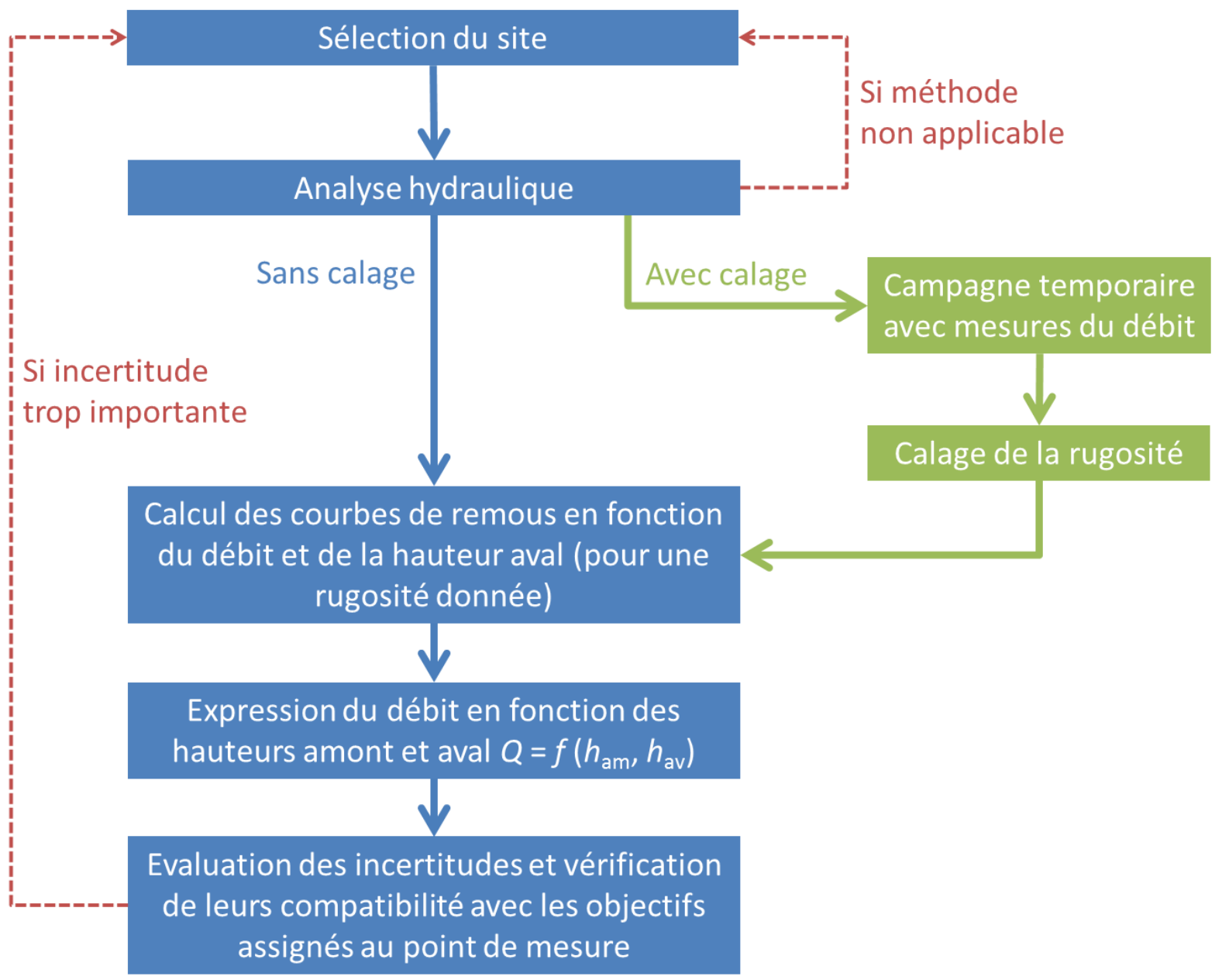

Figure 2. Logigramme de mise en œuvre de la méthode h2Q sur le terrain.

\subsection{Sélection du site}

Cette étape consiste à sélectionner le site de mesure, autrement dit les deux regards dans lesquels mettre en place les capteurs de niveaux, et à collecter l'ensemble des données d'entrée nécessaires à la méthode : forme et taille du collecteur, pente de la canalisation, distance entre les deux regards, gamme de débits à mesurer... La hauteur maximale susceptible d'être observée à l'aval est une information essentielle à connaître pour mener l'analyse hydraulique et évaluer l'adéquation de la méthode au site A ce stade, il est également important de vérifier qu'aucun apport de débit n'a lieu entre les deux regards et qu'aucune singularité n'est susceptible d'impacter la hauteur d'eau à proximité des points de mesure.

\subsection{Analyse hydraulique}

L'analyse hydraulique consiste à calculer les courbes de remous pour les débits minimum et maximum à mesurer, avec les hauteurs aval minimum et maximum susceptibles d'être observées. Il faut alors s'assurer que la différence de niveau entre les deux capteurs sera suffisamment importante (au moins $5 \mathrm{~cm}$ ) pour que la méthode soit précise. Si celle-ci n'est pas suffisante, il convient d'éloigner les deux 
capteurs. Le tableau suivant fournit quelques ordres de grandeur de la distance entre les deux capteurs nécessaire pour obtenir une mesure précise ; ils ont été calculés en considérant deux cas de figures : un débit de type temps sec (20\% de remplissage) et une influence aval égale à $50 \%$ du diamètre, un débit de type temps de pluie (50\% de remplissage) et une influence aval égale à $80 \%$ du diamètre. La distance donnée dans le tableau correspond au cas le plus défavorable. Ces distances ne constituent que des ordres de grandeur qui doivent être ajustés au cas par cas en fonction de l'analyse hydraulique; une distance inférieure ou supérieure peut être requise selon les sollicitations hydrauliques du collecteur (débit, hauteur).

\begin{tabular}{cccc}
\hline Pente & DN300 & DN500 & DN1000 \\
\hline $\mathbf{0 . 2 \%}$ & $70 \mathrm{~m}$ & $100 \mathrm{~m}$ & $170 \mathrm{~m}$ \\
$\mathbf{0 . 5 \%}$ & $30 \mathrm{~m}$ & $40 \mathrm{~m}$ & $70 \mathrm{~m}$ \\
\hline
\end{tabular}

Tableau 1. Ordres de grandeur de la distance nécessaire entre les deux capteurs pour garantir une mesure précise par la méthode $\mathrm{h} 2 \mathrm{Q}$.

\subsection{Méthode h2Q}

Un outil numérique de calcul de courbe de remous (calcul 1D) est utilisé pour générer l'ensemble des cas hydrauliquement possibles. L'ensemble de la gamme de variation des débits $Q$ et des hauteurs aval $h_{2}$ sont pris en considération pour calculer les hauteurs $h_{1}$ correspondantes (par exemple : $100 \mathrm{Q}$ et 100 $\mathrm{h}_{2}$ ). Ce calcul aboutit à la constitution d'un tableau de 10000 lignes présentant l'ensemble des cas possibles. Ce tableau $\left(h_{1}, h_{2}, Q\right)$ peut être formalisé sous la forme d'une fonction $Q=f\left(h_{1}, h_{2}\right)$ directement implémentable dans un superviseur ou bien programmé dans un tableur. Une courbe d'incertitude en fonction de la différence de niveau entre les deux regards est également fournie.

\subsection{Calage du coefficient de rugosité}

Pour calibrer la valeur de la rugosité, une mesure de référence doit être mise en place. Cette mesure doit avoir une incertitude suffisamment faible pour pouvoir être utilisée comme étalon. Pour cette mesure de référence, la méthode de mesure par dilution (par exemple un traçage à la rhodamine) est idéale dans la mesure où cette méthode présente une incertitude très faible, de l'ordre de $5 \%$ (Lepot et al 2014). Un jaugeage de la vitesse dans la section de passage de l'écoulement (par exemple par le biais d'un profilomètre) est également une méthode pertinente pour cette calibration. Enfin, le calage sur un enregistrement issu d'une campagne de mesure temporaire est également possible, à condition de s'assurer de la qualité de cette mesure.

Une fois ce calage effectué, la méthode peut être appliquée telle que décrite précédemment dans la partie 2.4.

\section{Exemples d'application et validation de la méthode}

Après avoir été validée dans le canal expérimental du hall hydraulique du laboratoire ICube (Isel 2014), la méthode a été mise en œuvre sur le terrain afin de vérifier son caractère opérationnel en réseau d'assainissement. Sept sites ont ainsi fait l'objet d'une mise en œuvre de la méthode h2Q et d'une confrontation à une mesure indépendante du débit. Deux exemples représentatifs sont détaillés ci-dessous.

- Un collecteur circulaire de diamètre $700 \mathrm{~mm}$ et présentant une pente de $0.34 \%$.

- Un collecteur ovoïde T200 présentant une pente de $0.05 \%$.

\subsection{Application $\mathrm{n}^{\circ} 1$}




\subsubsection{Description du site et de l'instrumentation}

Le collecteur est une canalisation circulaire de diamètre $700 \mathrm{~mm}$. Elle présente une pente de $0.34 \%$. Les deux capteurs de niveau d'eau ont été mis en place dans deux regards distants de $134 \mathrm{~m}$. Un profilomètre a été posé dans un regard intermédiaire afin de disposer d'une mesure du débit pour évaluer la performance de la méthode h2Q. Cette mesure est de très bonne qualité et on peut estimer sa précision à environ $10 \%$. La comparaison a été effectuée sur une période de deux semaines (du 11 au 25/07/2014).

\subsubsection{Mise en cuvre de la méthode h2Q sans calage}

La méthode h2Q a été mise en œuvre sans calage particulier sur ce site. Les résultats ont été comparés aux débits fournis par le profilomètre. La figure 3 fournit une vue de cette comparaison sur la période allant du 16 au 24/07/2014. On y constate une très bonne concordance entre la méthode h2Q et les mesures du profilomètre. Ce constat est valable aussi bien en temps sec (journées du 16 au 20/07/2014) qu'en temps de pluie (journées du 21 au 23/07/2014). La figure 4 montre quant à elle le débit mesuré par le profilomètre en fonction du débit calculé selon la méthode $\mathrm{h} 2 \mathrm{Q}$. Sur la totalité des deux semaines, l'écart moyen est de $10 \%$. Par ailleurs $80 \%$ des débits mesurés avec la méthode h2Q ont un écart avec le débit mesuré par le profilomètre inférieur à $16 \%$. Seuls quelques points s'écartent de la première bissectrice (notamment entre 200 et 250 l/s) ; cet écart reste cependant très limité. Les résultats étant déjà très bons, aucun calage n'a été effectué sur ce site.

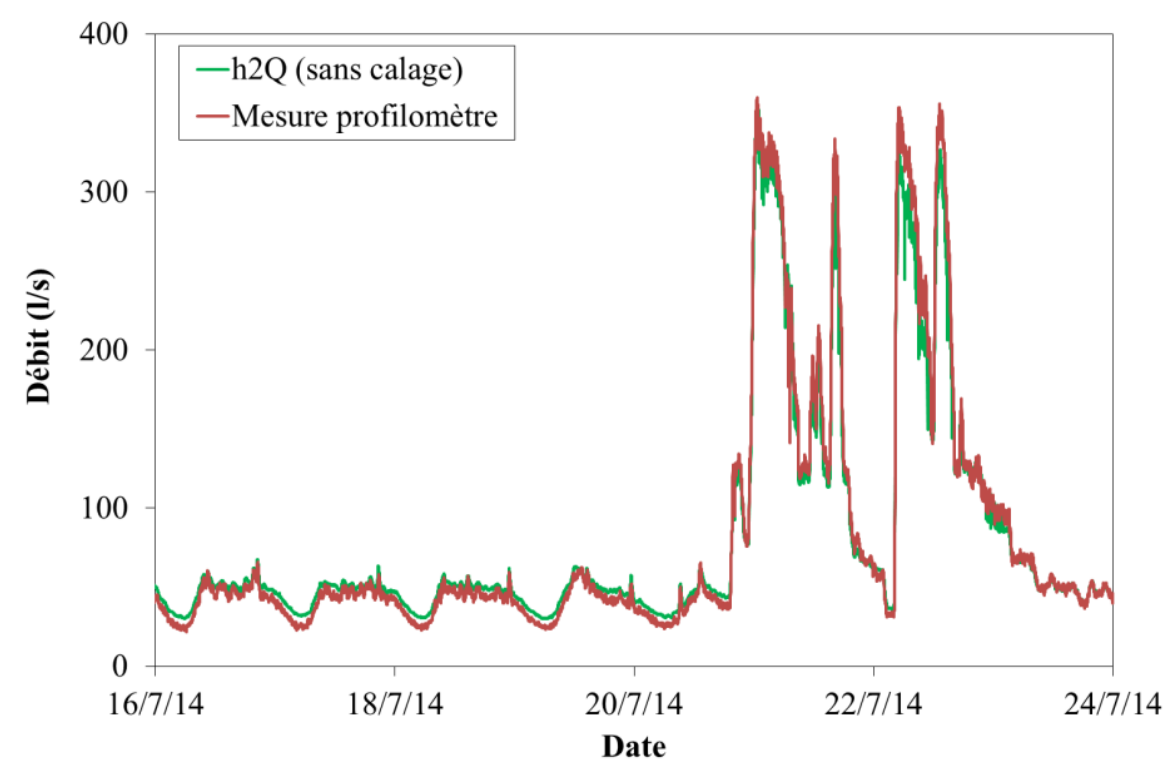

Figure 3. Comparaison entre la méthode h2Q (sans calage) et la mesure par profilomètre sur le site d'application $\mathrm{n}^{\circ} 1$ entre le 16 et le 24 juillet 2014 . 


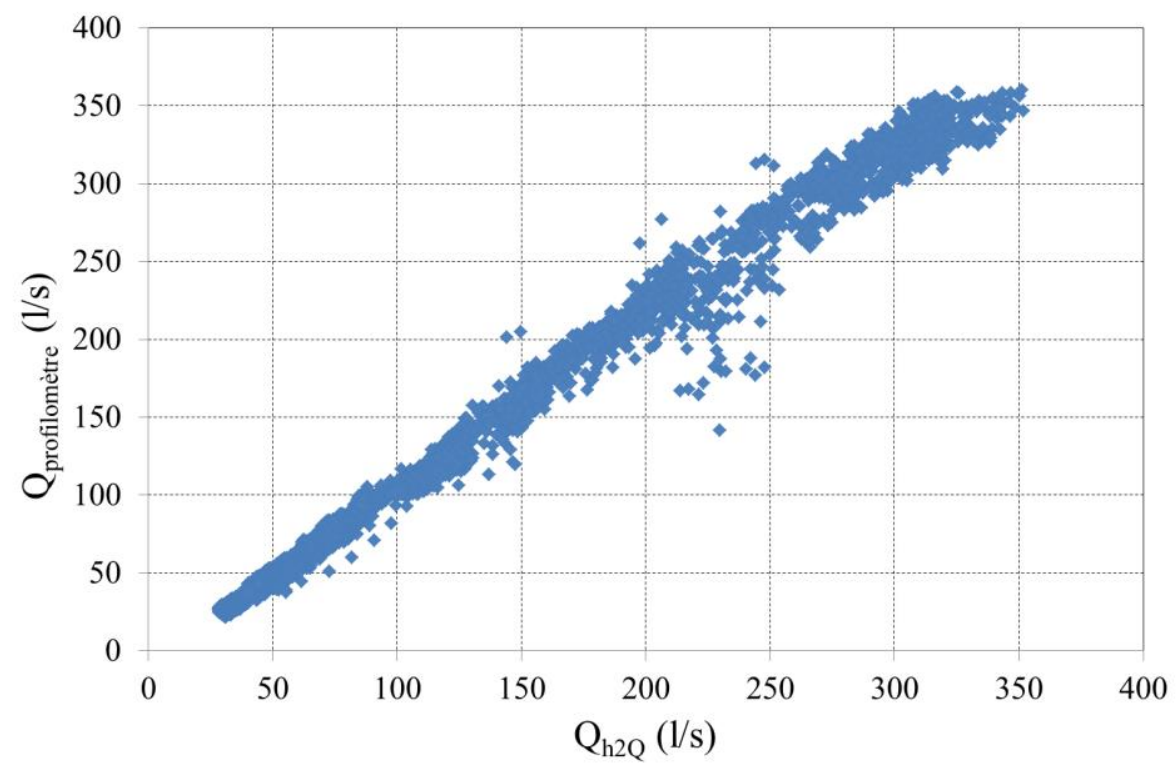

Figure 4. Comparaison entre le débit mesuré par profilomètre et le débit calculé selon la méthode h2Q

A titre de comparaison, 1'utilisation d'une relation de Manning avec la pente du radier conduirait à une surestimation de l'ordre de $25 \%$, et l'utilisation de la pente moyenne de la ligne d'eau réduit la dispersion des résultats, mais par le biais.

\subsection{Application $n^{\circ} 2$}

\subsubsection{Description du site et de l'instrumentation}

Le collecteur instrumenté est un ovoïde T200 présentant une pente très faible de $0.05 \%$. Ce site est déjà équipé par ailleurs d'un capteur Doppler et d'une corde de vitesse donnant des résultats cohérents entre eux; ces deux mesures indépendantes serviront de support de comparaison des débits déterminées avec la méthode h2Q. Compte tenu de la qualité de ces mesures et surtout de leur redondance, on estime que le débit est connu avec une précision de l'ordre de $20 \%$.

Compte tenu de la grande taille du collecteur et afin d'avoir une différence de niveau significative entre les deux regards (et donc une incertitude de mesure faible), les deux capteurs de niveau ont été mis en place dans deux regards distants de 350 mètres. Il a été vérifié qu'aucun apport intermédiaire de débit n'avait lieu sur cette distance. La méthode h2Q a ensuite été mise en œuvre dans un premier temps sans calage puis avec calage. Ces deux mises en œuvre différentes permettent de quantifier le gain d'un calage de la méthode sur la précision des résultats. Les comparaisons ont été effectuées sur une période d'environ trois mois (du 20/11/2014 au 18/02/2015). Dans un objectif de clarté, les graphiques présentées ci-dessous correspondent à seulement quelques jours de cette période.

\subsubsection{Mise en æuvve de la méthode h2Q sans calage}

Les résultats obtenus avec la méthode h2Q sans calage sont illustrés pour une huitaine de jours sur la figure 5. Sur ces quelques jours, on trouve quelques jours de temps sec où le débit fluctue entre environ 20 et 160 1/s (du 7 au 12/12/2014) et deux jours de temps de pluie où le débit monte jusqu'à quasiment 500 1/s (12 et 13/12/2014). Ces deux types d'évènements sont bien reproduits en termes de dynamique par la méthode h2Q sans calage, la figure 4 mettant en effet une bonne concordance des fluctuations de débits ainsi que des pics entre la méthode h2Q sans calage et la mesure Doppler. 
Quantitativement, on constate cependant des différences, la méthode h2Q sous-estimant le débit en moyenne de $17 \%$ par rapport à la mesure Doppler.

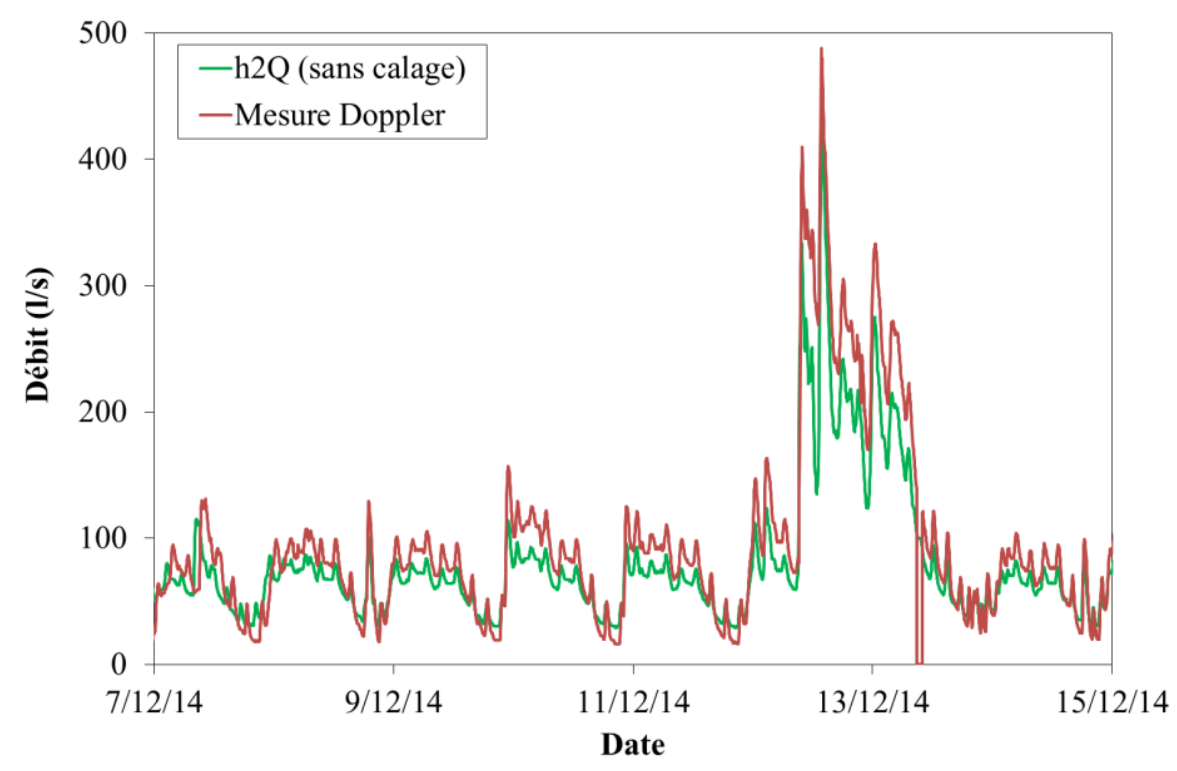

Figure 5. Comparaison entre la méthode h2Q (sans calage) et la mesure Doppler entre le 7 et le 15 décembre 2014 pour le site d'application $\mathrm{n}^{\circ} 2$.

\subsubsection{Mise en cuvre de la méthode h2Q avec calage}

A titre d'exercice la méthode $\mathrm{h} 2 \mathrm{Q}$ a été calibrée pour vérifier la faisabilité d'une correction de biais et à quantifier le gain potentiel d'une telle approche. La rugosité de Strickler a ainsi été calibrée à une valeur de $81 \mathrm{~m}^{1 / 3} / \mathrm{s}$. Cette valeur est significativement différente de la valeur de $70 \mathrm{~m}^{1 / 3} / \mathrm{s}$ considérée par défaut mais elle demeure cohérente avec la rugosité d'une canalisation d'assainissement. La figure 6 illustre les résultats obtenus avec la méthode calée. A présent, l'écart moyen entre la méthode h2Q et la mesure Doppler est inférieur à 5\% : la précision de la méthode est désormais voisine de celle du Doppler, mais celle-ci n'a pas été évaluée pour ce site. La huitaine de jours représentés sur la figure 5 illustre cette comparaison pour quelques journées de temps sec et une pluie (même période que la figure 4).

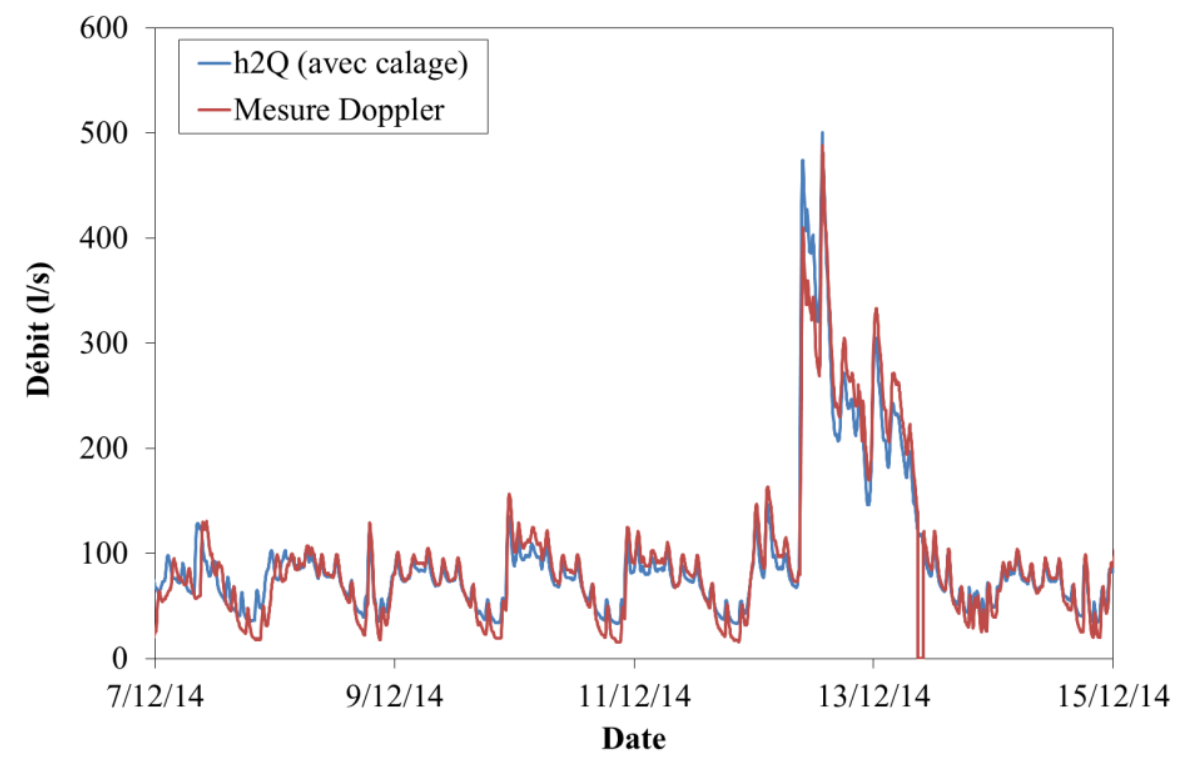


Figure 6. Comparaison entre la méthode h2Q (avec calage) et la mesure Doppler entre le 7 et le 15 décembre 2014 pour le site d'application $\mathrm{n}^{\circ} 2$.

\section{Conclusion}

Amélioration de la méthode présentée dans la Charte qualité de l'hydrométrie, la méthode h2Q a depuis été mise en œuvre sur plusieurs sites grandeur nature sur lesquels elle a démontré son caractère opérationnel. En reposant uniquement sur la mesure de hauteurs d'eau à l'aide de capteurs sans contact, cette méthode se caractérise par des coûts d'investissement et de maintenance peu importants.

Mise en œuvre sans calage, la méthode h2Q permet une évaluation du débit avec une incertitude de l'ordre de 30\%. Si cette incertitude est jugée trop importante, la méthode peut être mise en œuvre avec calage sur une mesure de référence du débit qui doit bien évidemment être précise (dilution, profilomètre...). Dans ce cas, elle permet l'évaluation du débit avec une incertitude de l'ordre de l'ordre de $15 \%$, ce qui est comparable aux autres technologies disponibles sur le marché lorsqu'elles sont mises en place dans de bonnes conditions.

Deux applications sur site ont été présentées : la première sur un collecteur de grande taille (ovoïde T200), la seconde sur un collecteur de taille moyenne (circulaire $700 \mathrm{~mm}$ ). Dans les deux cas, la méthode h2Q s'est révélée être en très bon accord avec les mesures alternatives du débit mises en place afin de valider la méthode. Une mise en œuvre sur des collecteurs de plus petite taille (DN300, DN400...) est également possible, pourvu que les conditions d'application de la méthode soient respectées (pas de singularité ni d'apport latéral entre les deux regards, différence de niveau d'eau entre les deux capteurs d'au moins $5 \mathrm{~cm}$ ).

\section{Remerciements}

Le projet MENTOR a bénéficié du soutien financier de l'Agence Nationale de la Recherche, « Notification de décision d'aide ${ }^{\circ}$ ANR 11 ECOT 00701 du 13 décembre $2011 »$.

\section{Références}

Hager W H (1999). Wastewater hydraulics - Theory and practice. Springer.

Isel S (2014). Développement de méthodologies et d'outils numériques pour l'évaluation du débit en ;réseau hydraulique à surface libre. Thèse de doctorat, Université de Strasbourg.

Isel S, Araud Q, Vazquez J, Dufresne M (2014). Développement d'outils et de méthodologies pour déterminer le débit par mesures de hauteurs d'eau en collecteur de réseau d'assainissement. Techniques Sciences Méthodes 1-2:71-79.

Isel S, Vazquez J, Dufresne M, Fischer M, Joannis C (2016). Méthode h2Q : Une méthode innovante pour évaluer le débit en collecteur par la mesure de deux hauteurs d'eau. Livrable du projet Mentor (Agence Nationale de la Recherche).

Lepot M, Momplot A, Lipeme Kouyi G, Bertrand-Krajewski J-L (2014). Rhodamine WT tracer experiments to check flow measurements in sewers. Flow Measurement and Instrumentation, 40:2838.

ISO (2004). Hydrometry - Measurement of discharge by the ultrasonic (acoustic) method, International Standardization Association, ISO 6416:2004. 
ISO (2010). Hydrometry - Guidelines for the application of acoustic velocity meters using the Doppler and echo correlation methods, International Standardization Association, 15769:2010.

Ministère de l'Environnement (1998). Charte qualité de l'hydrométrie - Code de bonnes pratiques. 